\title{
Hybrid Technique on Image Clustering
}

\section{Rakhi}

\begin{abstract}
Face recognition using FLD for extracting high dimensional images is introduced in this paper. The main purpose is to work on removing bugs and noise from the images and extract the facial expression applied on face descriptor. FLD is selected for increasing the discrimination information [17]. The main points of this paper give the brief knowledge about the face recognition and face clustering. Its shows how biometric terms help the local and global features for extracting information from database. Finding better solutions to deal with noise in face recognition is a challenging task [18]. We also performed some comparative analysis on various face recognition techniques. The main motive of this paper is to increase the recognition rate of the images and provide good efficiency. This method defines how the features and facial expression are extracted and all noise and bugs are eliminated to make a separate individual cluster of same known faces.
\end{abstract}

Keywords: Face Recognition, Biometric, Local and Global features, FLD, Face Clustering, Gabor Wavelet

\section{INTRODUCTION}

A face clustering is done by combining two parameters which are face recognition and face detection. Biometric patterns help in identification of different ratio between the aspects of our face. If we talk about the human brain it has strong capability to identify the different faces even having the small changes in human faces. It is so amazing how our brain can identify these changes. Face recognition is important in various field for solving the security issues. Over the past of 30-40 years the scientist had made the replica of neuron system of our brain which helps in the various face recognition technology, having the same property to identify the face just like our brain does. It is exceedingly difficult task to manage and identify the images and put it into the cluster in the real environment. We used face recognition techniques to increase the recognition rate. The idea for the technology we used today is inspired by our brain neuron and fuzzy etc. Basically face recognition has two parameters (1) verification in which the system determines whether the images actually relates to the individual and check into the database if it is not there then the request is rejected.(2) Identification in which the system check the pre-existing data from the database which identify the face if it is from known individuals or not[1]. Biometric is the approach which is used for face recognition having a different pattern like physical or behaviour characteristic. Biometric technique helps us to identify the character of individuals. Today, in the real world there are lot of data and faces which is hard to recognize in one go and make a cluster of individuals.

Revised Manuscript Received on August 15, 2020.

* Correspondence Author

Rakhi*, Deenbandhu Chhotu Ram University Science and Technology, Murthal, Sonipat (Haryana), India. E-mail: Sharma.rakhi1641@gmail.com

(C) The Authors. Published by Blue Eyes Intelligence Engineering and Sciences Publication (BEIESP). This is an open access article under the CC BY-NC-ND license (http://creativecommons.org/licenses/by-nc-nd/4.0/)
Where the biometric technique help us to reliance and provide the security to the user where his/her data is confidential as in system after the scanning of eyes and fingerprint the system shuts off automatically within the seconds as per there authentication is stay within that particular user. According to the recent survey "ping identify survey" the security of accessing the data of the user is about $92 \%$. One of biometric approach is physical characteristics in which the recognition of the user is identified by his body parts just like eyes, nose, chin, voice, fingerprints, iris, photos and DNA etc. Face recognition is done by matching the photos from the existing databases to check if it is present in the database or not. Biometric recognizes the faces and determine the difference in different faces using some landmarks these landmarks are defined by the VISIONICS as known as nodal points of face which are about 80 in number. These nodal points help the system to identify the difference between the faces e.g.: the distance between the eyes, the depth of eyes, length and width of the nose, cheekbones, area of eyelids, the shape and size of lips etc. The second characteristic of biometric approach is behavioural in which the individual is identified by his behaviour like style of typing, engagement patterns, physical movements etc. After the identification of faces it matches from the existing database which is known as face detection and according to recognition and detection of faces, the system put similar known individuals in one group and another individuals in another group that's how the cluster is made.

\section{A. Face Recognition}

Now a days, it is very hard to recognize the person from large data sets, thanks to this various face recognition algorithms which help us to provide the various terms such as identification of the person, security issues, verifying the claims and providing the authentication of individual etc. There are three main parts of recognizing the data as shown in following figure.

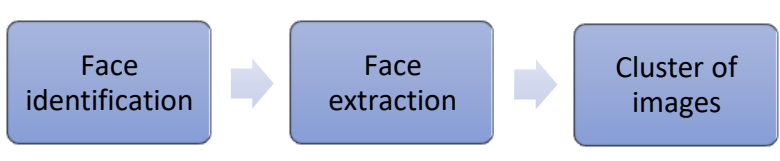

Fig 1: Face Recognition Steps

For making the perfect group of images we basically used three stages (1) face identification in which the images go under pre-processing and detection of various components of the images having different aspects and values which is faded due to noise and illumination. After this it also remove the unwanted material from the images and focus only on the face.

Published By:

Blue Eyes Intelligence Engineering

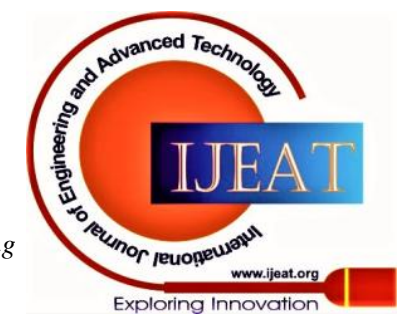




\section{Hybrid Technique on Image Clustering}

To detect the various components in the image biometric term is used to detect the various landmarks of the faces. Where the system detects the images and identifies from the existing database. (2) Face extraction is used for increasing the efficiency and recognition rate of the image. It is very crucial stage among all the three stages because in this stage we need the accurate result of the image to process it. Jyotikumari et al.[2] talked about the gabor filter technique which is very popular for face extraction in the images. More techniques like local binary pattern (LBP)[3], principle component analysis (PCA)[4] and linear discriminant analysis (LDA)[5] etc are also very popular and used for extracting the facial characteristics.(3) Cluster of images in which classifier play the important role. Classifier categorize the images which is extracted from the previous stage and make a group of the similar data known as cluster of data. The most popular classifier for order the image is support vector machine (SVM)[6].

\section{LITERATURE REVIEW}

This section discusses the previous work done in face recognition techniques.

[7]xiao han et. al direct the convolution neural network (CNN) to achieve the face recognition with good efficiency. It gives the brief insight about the deep learning, face recognition and convolution neural network. The motivation of this paper is used to increase the recognition rate of the faces. It identifies the covNet with the help of DBS framework which work on low level having the complex interaction within the stages. The main centre is to study the depth in learning for face recognition in biometric. For achieving this LFW database is used for increasing the recognition rate of the face. It overcomes the limitation of complex interaction of image having different aspects and features which gives the accurate figure about deep learning.

[8]Anil $\mathbf{J}$ et. al gives the good knowledge about the FER (face expression recognition) and face recognition. In this he used pre-existing algorithm for recognition like curvelet features extraction, gradient features matching and patch geodesic texture transformation. All these techniques are used for improving the efficiency rate of the image. This paper also shows the accuracy result of each technique individually. Both local and holistic features give the high recognition rate and enormous result as both are hybrid techniques.

[9]shallu sharma el. at compares the various pooling strategies and determine the differentiate between some pooling strategies like SPP(spatial pyramid pooling), stochastic pooling, rank based pooling, gated max average pooling, average pooling and tree pooling. This paper also gives the brief about deep learning, feed forward neural network with architecture and CNN and latest strategies. These pooling methods are used for the purpose of solving computer vision problem and conclude the invariant features and reduce outfitting work on the database MNIST, SVHN etc.

[10]Dipesh Vaya el. at attempts to increase the accuracy using PCA(principle component analysis). Author also gives the good knowledge about the face recognition and PCA. Manhattan distance method is used to determine the distance between the points and results are shown in table having eigenfaces approach which help in squeezing the duplication data.

[11]Yu Cheng el. at purposed imaged sequence clustering algorithm and gives the brief about face image clustering, biometric and face classifier and face detection. The author shows the skin segmentation using normalization which is used for transferring the data into the specific size. For detecting the various faces from database, he used single gussian model. The main purpose of this paper is to increase the accuracy and precision rate of the images and make a proper cluster of it.

[12]Shraddha Arya el. at purposed the new model LDA(linear discrimination analysis) for recognition of faces which gives the better efficiency and extract the features from images. Author used the neural network technique to train the data and the database is taken as random images from all the direction like right, left and front etc and process it into three phases (1)train the data (2)convert it into the phase (3)recognize the face. This paper also gives the good knowledge about the facial features, LDA appearance and deep learning.

[13]Chuanzhen Rong el. at gives the brief on human face, face detection and colour space. The author attempts to increase the efficiency of the image and detect the system using the SPC(spatial pattern clustering) algorithm where every pixel is used to determine the face colour as pattern which shows the information and features and then make a cluster of it. To determine the distance between the pixel in images the author used geodesic distance to achieve the increase in efficiency. This paper shows the dissimilarities measurement and determine the weight factors of each pixels.

\section{A. Comparative analysis}

The literature review presented in previous section is analyzed in the table below.

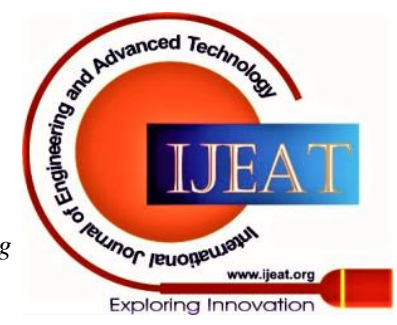


Table 1: Comparative Analysis

\begin{tabular}{|c|c|c|c|c|}
\hline $\begin{array}{l}\text { Year of } \\
\text { publica } \\
\text { tion }\end{array}$ & $\begin{array}{l}\text { Author's } \\
\text { name }\end{array}$ & $\begin{array}{l}\text { Algorithm and } \\
\text { method used }\end{array}$ & Advantages & Disadvantages \\
\hline 2018 & $\begin{array}{l}\text { xiao han } \\
\text { et. At }\end{array}$ & $\begin{array}{l}\text { CNN with neural } \\
\text { network }\end{array}$ & $\begin{array}{l}\text { Simple, fast and effectively } \\
\text { detect the faces. }\end{array}$ & $\begin{array}{l}\text { Parameter setting of multi } \\
\text { hidden layer is not solved. }\end{array}$ \\
\hline 2016 & $\begin{array}{l}\text { Anil J et. } \\
\text { At }\end{array}$ & $\begin{array}{l}\text { FER, FARO 3-D face } \\
\text { recognition, Curvelet } \\
\text { Feature Extraction } \\
\text { and gradient feature } \\
\text { matching }\end{array}$ & $\begin{array}{l}\text { Removing of bugs and } \\
\text { increase the recognition rate. }\end{array}$ & Technique is quite old. \\
\hline 2019 & $\begin{array}{c}\text { shallu } \\
\text { sharma el. } \\
\text { At }\end{array}$ & $\begin{array}{l}\text { CNN with pooling } \\
\text { strategy }\end{array}$ & $\begin{array}{l}\text { Solve the problem of } \\
\text { computer vision and } \\
\text { overfitting. }\end{array}$ & $\begin{array}{l}\text { Pooling method relies on } \\
\text { architecture. }\end{array}$ \\
\hline 2018 & $\begin{array}{c}\text { Dipesh } \\
\text { Vaya el. At }\end{array}$ & $\begin{array}{c}\text { PCA (Principle } \\
\text { Component analysis) }\end{array}$ & $\begin{array}{l}\text { Increase the accuracy and } \\
\text { eliminate duplicate data. }\end{array}$ & $\begin{array}{c}\text { Classification take time and } \\
\text { complex mathematical } \\
\text { equation. }\end{array}$ \\
\hline 2009 & $\begin{array}{l}\text { Yu Cheng } \\
\text { el. At }\end{array}$ & $\begin{array}{l}\text { Image sequence } \\
\text { clustering }\end{array}$ & $\begin{array}{l}\text { Increase the precision rate } \\
\text { and convert the images in } \\
\text { specific size. }\end{array}$ & $\begin{array}{l}\text { Illumination effect is not } \\
\text { reduced. }\end{array}$ \\
\hline 2018 & $\begin{array}{c}\text { Shraddha } \\
\text { Arya el. At }\end{array}$ & $\begin{array}{l}\text { LDA(linear } \\
\text { discrimination } \\
\text { analysis) }\end{array}$ & $\begin{array}{l}\text { Increase the efficiency and } \\
\text { feature of images. }\end{array}$ & $\begin{array}{l}\text { Training of neural network } \\
\text { is not up to the mark. }\end{array}$ \\
\hline 2015 & $\begin{array}{c}\text { Chuanzhen } \\
\text { Rong el. } \\
\text { At }\end{array}$ & $\begin{array}{c}\text { SPC } \\
\text { algorithm(spatial } \\
\text { partial clustering) }\end{array}$ & $\begin{array}{l}\text { Improve the clustering } \\
\text { method. }\end{array}$ & $\begin{array}{l}\text { Pixel weight factor is so } \\
\text { complex. }\end{array}$ \\
\hline
\end{tabular}

\section{TECHNIQUE OF FACE CLUSTERING}

Face recognition is used for extracting the features and converting it into the high dimensional images. It shows the various entity of images to access the best come out from it. To detect exact dimensions of the faces, system check the scale of each images from close range so, local and global features having some property through which system make our output so reliable. The local feature mainly focuses on the edge, corner, lines and curve of the image on the other hand global feature focus on the dimensions, frequency, matrix and statistics of the image. It uses vector in featuring of image to reduce the robustness, illumination and secure this from changing the scale of the images. Both local and global have the different property which shows the best image formation and at the end gives the best efficient image. These are the main steps for recognizing the face. First, we take out the raw data from our selected database and system recognize it partially as per their property where both the features extract the image and retrieve the meaningful information for making our result reliable. Both local and global features work differently where local features works only on some points whereas the global feature works on whole image at once as explained in following image[14].

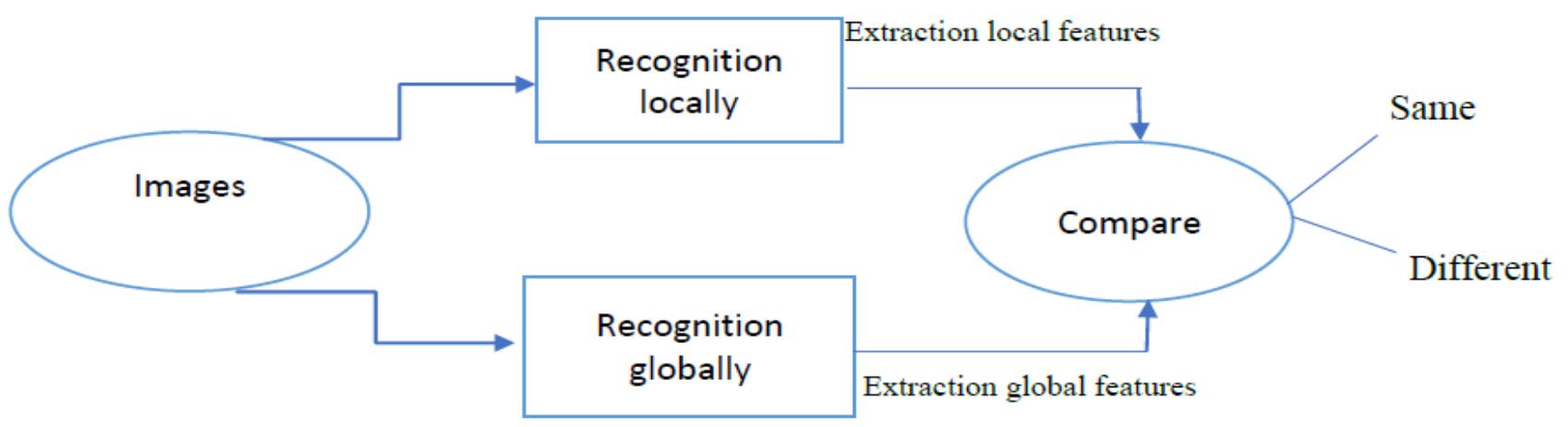

Fig 2: local and global face extraction

\section{A. Feature descriptor}

Feature descriptor is main term which is used for extracting the useful information of the images. It increases the robustness of the image on set of vectors. It takes out the IPs and shows the feature of images and detect the various bugs which may harmful for our result. The purposed approach for extracting the efficient data uses both local and global feature in wavelet transformation using fisher linear analysis.

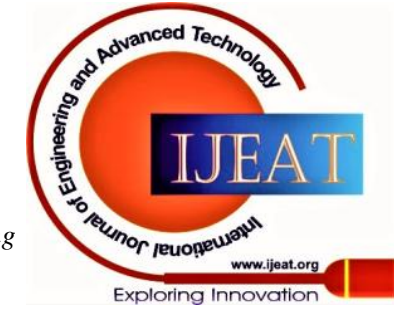




\section{Hybrid Technique on Image Clustering}

The main motive of this approach is to increase the performance rate and recognition rate of the image.

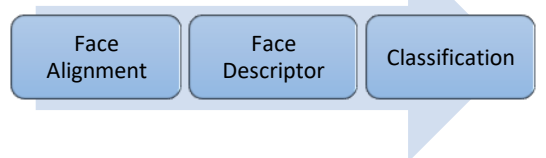

As we can see from above figure that the raw images are firstly processed from database in face alignment to determine the structure of the face digitally in manner. This is the technology which automatically determine the nodal points of the faces such as eyes, nose, lips etc. Secondly the face descriptor is used for removing all the noise and unwanted bug from the images. At last classification is classified for all the extracted images into systematic arrangement just like we divide the categories some time. That's how we get the cluster of images having the same individual into single folder and other individual into another folder then cluster is made with good efficiency and in less amount of time.

\section{B. Local feature extraction using gabor wavelet}

The gabor wavelet used to pull out the information from faces. GWT is used for extracting the facial expression of faces. From recent study we

know that if we use only global feature for extracting the images it give us some disadvantages like noise, issue in measuring landmarks of face and scaling issues will occur that's why we purpose the both local and global feature using wavelet transformation. Gabor help us to improve the frequency of images and analysis of the specific content. It takes a wide quantity of systems with numerous scales and route. Gabor easily detect the landmarks of faces and characterize into specified image. Kim et al (2015) proposed a joined subspace approach utilizing both worldwide and community highlights for face confirmation. LDA approach highlights the near pixels globally in which the vector of each image is analysed by LGFV local gabor feature vector. Gabor wavelet put in neighbourhood factors of describing them. Gabor wavelet mainly aim to highlight on extraction of different points of the face. To identify the features gabor used the gabor amplitude and multi-frequency for detecting the facial expressions, smaller change in position may change the entire layout of the images which is recovered from neighbour data and select the facial expression from sub-images[15].

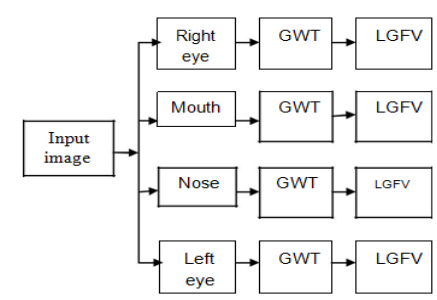

\section{FISHER LINEAR DISCRIMINANT (FLD)}

Fisher Linear Discriminant model for improving the capability of generalization of classifier and also enforced to solve the problem of face recognition[16]. When the over- fitting occurs its decrease the noise in data. It is used to minimize the variance and in statistically manner it maximizes the distance between the class's mean. The main function of FLD is used to determine the classification of data.

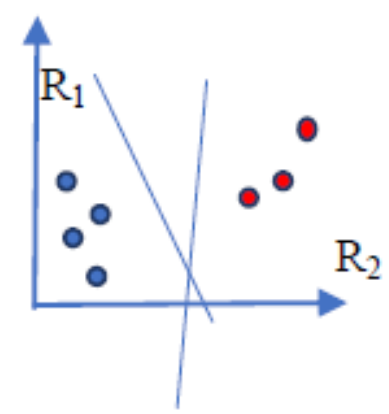

This basic structure shows the proper group division between two classes shown on $\mathrm{X}$ and $\mathrm{Y}$ line name as $\mathrm{R}_{1}$ and $R_{2}$. FLD does the final classification between the individuals. It helps the system to show the exact difference between the two images and divides into proper cluster having the same entity in one group as so on. Classified data is used to make a proper band of image. It is very hard to put the data in perfect ration so it help us to convert the high dimensional image into the one - dimensional image for making groups because detecting high dimensional images is not easy as one line data. To make a cluster of the image data should be in one- dimensional. For testing we take AR database, Google faces and random images to analyse the quality of an image. Normal image is highlighted to one dimensional image with the help of vector in vector section. The difference between local and global features shows in given table and graph. It is observed that the usage of both the local and global feature combinations lead to higher recognition rate.

\begin{tabular}{|l|l|l|l|}
\hline \multicolumn{3}{|c|}{ Recognition rate } & FAR \\
\hline $\begin{array}{l}\text { Local } \\
\text { feature } \\
\text { only }\end{array}$ & $\begin{array}{l}\text { Global } \\
\text { feature } \\
\text { only }\end{array}$ & $\begin{array}{l}\text { Both local and } \\
\text { global feature }\end{array}$ & \\
\hline 0.55 & 0.75 & 0.86 & 1 \\
\hline 0.6 & 0.8 & 0.9 & 2 \\
\hline 0.65 & 0.81 & 0.95 & 3 \\
\hline 0.7 & 0.83 & 0.96 & 4 \\
\hline
\end{tabular}

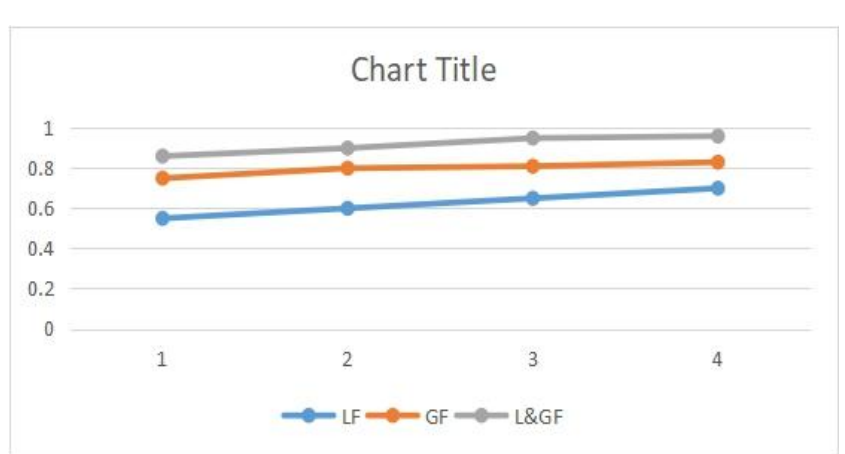

This database incorporates 400 pictures of subject and 10 cluster of images having the resolution rate 112.92 . It is also used to increase the MDF (most discriminating features) and solve the heavy number of differentiate between the classes.

Published By:

Blue Eyes Intelligence Engineering

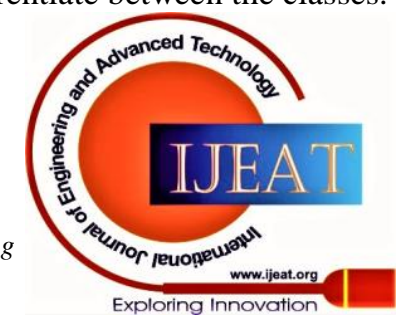


LD is implemented with local extraction and global extraction cutting edge quality to approve the characteristics. FLD is used for installation of each vector as fragment classifier where each segment vector encode the record of uneven organization and make a cluster of it.

\section{CONCLUSION \& FUTURE WORK}

It presents the novel technique FDT having local and global feature extraction using gabor wavelet for face clustering. Based on cut off parameters it creates a fundamental result which shows increment in recognition rate as compared to the rate if we used the local and global features separately. Above used database is trapped of react with different parameters. Gabor is used to show the difference of images like scaling, expressions etc. FDT is used for making classification of extracted data. Our next goal will be auditing the perquisite pairs and checks the relation between multi-interface in order to improve the collection. Especially if we used numerously ex: semilabeled datasets and face from video and others.

\section{REFERENCES}

1. Kittikhun Meethongjan \& Dzulkifli Mohamad "A Summary of literature review : Face Recognition" Postgraduate Annual Research Seminar 2007 (3-4 July 2007)"

2. Jyoti Kumari, R.Rajesh, KM.Pooja, "Facial expression recognition: A survey", Second International Symposium on Computer Vision and the Internet, Procedia Computer Science 58 ( 2015 ) 486 - 491

3. Ojala T, Pietik"ainen M, Harwood D. "A comparative study of texture measures with classification based on featured distributions", Pattern recognition 1996;29:51-9

4. Turk MA, Pentland AP. "Face recognition using eigenfaces. In: Computer Vision and Pattern Recognition", Proceedings, CVPR'91 IEEE Computer Society Conference on. IEEE; 1991, p. 586-91.

5. Belhumeur PN, Hespanha JP, Kriegman D. Eigenfaces vs. fisherfaces: Recognition using class specific linear projection. Pattern Analysis and Machine Intelligence, IEEE Transactions on 1997;19:711-20.

6. Hsu CW, Chang CC, Lin CJ, et al. "A practical guide to support vector classification". 2003

7. Xiao Han \& Qingdong Du, "Research on Face Recognition Based on Deep Learning",

8. Anil j and Dr. L. Padma Suresh "Literature survey on Face and Face Expression

9. Recognition", 2016 International Conference on Circuit, Power and Computing Technologies [ICCPCT]

10. Shallu Sharma and Rajesh Mehra, "Implications of Pooling Strategies in Convolutional Neural Networks: A Deep Insight"

11. Dipesh Vaya and Teena Hadpawat, "PCA BASED EFFICIENT FACE RECOGNITION TECHNIQUE”, International Journal For Technological Research In Engineering ISSN (Online): 2347 - 4718

12. Yu Cheng, Tao Zhang and Song Chen, "Fast Person-Specific Image Retrieval Using a Simple and Efficient Clustering Method",International Conference on Robotics and Biomimetics December 19 -23, 2009

13. Shraddha Arya and Arpit Agarwal, "Face Recognition with partial Face Recognition and convolutional neural network", international journal of advance research in computer engineering \& technology(IJARCET), issue 1 January, 2018

14. Chuanzhen Rong, Yongxing Jia, Yu Yang, Ying Zhu, Yuan Wang, Xue Ni, "Face Detection Based on Improved Clustering of Spatial Patterns", 2015 8th International Congress on Image and Signal Processing

15. Leila Kabbai, Mehrez Abdellaoui, Ali Douik, "Image classifification by combining local and global features", (C) Springer-Verlag GmbH Germany, part of Springer Nature 2018

16. SHI DongCheng, CAI Fang, DU Guangyi, "Facial Expression Recognition Based on Gabor Wavelet Phase Features", 2013 IEEE

17. Chengjun Liu and Harry Wechsler, "Enhanced Fisher Linear Discriminant Models for Face Recognition"

18. Zhihua Xie, Guodong Liu, Shiqian Wu, Zhijun Fang, "Infrared Face Recognition Based on Blood Perfusion and Fisher Linear
Discrimination Analysis", International Workshop on Imaging Systems and Techniques, May 11, 2009

19. Suman, Parvinder Singh, R. B. Patel, "Intelligent and Secure Routing in Heterogeneous Wireless Networks", Proceedings of the International Conference on Wireless Networks (ICWN), 2014

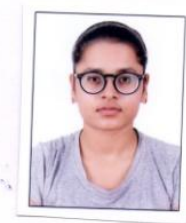

\section{AUTHOR'S PROFILE}

I'm Rakhi graduated from Bhagat Phool Singh Mahila Vishwavidhyla khanpur and pursing my Post Graduation from Deenbandhu Chhotu Ram University Science and Technology Murthal Sonipat (Haryana). I did my Schooling from Saraswati Shiksha Sanathn High school and I did my diploma from Government Polytechnique Sonipat (Haryana) e-mail: Sharma.rakhi1641@gmail.com Address: H. No. 1641, sec- 15, Old Housing Board Colony Sonipat (Hryana), India. Ph. No: +91-9467371646

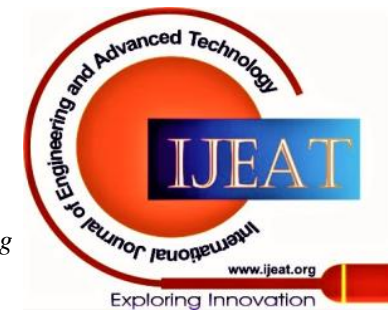

